Journal of the Mathematical Society of Japan Vol. 3, No. 1, May, 1951.

\title{
Zur Geschlechtertheorie in quadratischen Zahlkörpern
}

\section{Helmut Hasse}

Die Theorie der Geschlechter in quadratischen Zahlkörpern wurdeals Theorie der Geschlechter quadratischer Formen - von Gauss in seinen Disquisitiones Arithmeticae entwickelt. Ihre Verallgemeinerung auf beliebige relativ-zyklische Zahlkörper von Primzahlgrad bildet ein wesentliches Hilfsmittel beim Aufbau der allgemeinen Klassenkörpertheorie, nämlich für den Beweis der dortigen grundlegenden Ungleichung $h \geqq n$ zwischen dem Grad $n$ des betrachteten zyklischen Relativkörpers und dem Index $h$ der ihm zugeordneten Divisorengruppe im Grundkörper. Dies gilt sowohl für den ursprünglichen Beweis von Takagi $i^{1)}$, als auch für den neuerdings auf veränderter (Grundlage gegebenen Beweis von Chevalley"), bei dem letzteren allerdings in mehr versteckter Gestalt als bei dem ersteren.

Die in diese Beweise eingehenden Schlüsse aus der Geschlechtertheorie haben im wesentlichen abzählenden Charakter. Der einfache begriffliche Kern der Theorie, die dort nur Hilfsmittel, nicht Selbstzweck ist, tritt bei ihnen gar nicht hervor. Ist man einmal im Besitz der Hauptsätze der Klassenkörpertheorie, so lässt sich die Geschlechtertheorie ganz einfach in durchsichtiger, rein begrifflicher Gestalt herausarbeiten. Das soll in dieser kurzen Note für den klassischen Spezialfall der quadratischen Zahlkörper geschehen.

Anlass zu dieser Note ist eine von Artin bemerkte Unrichtigkeit in einer früheren Arbeit von mir. ${ }^{3)}$ Ich komme darauf am Schluss zurück.

1. Sei $\mathrm{P}$ der rationale Zahlkörper, $\Omega=\mathrm{P}(\sqrt{ } \bar{d})$ ein quadratischer Zahlkörper mit der Diskriminante $d$, und $\mathrm{K}$ sein absoluter Klassenköper im engeren Sinne, d.h, der Gruppe $E$ aller Hauptdivisoren von $\Omega$ mit positiver Norm zugeordnet. Nach der Klassenïörpertheorie ist $K$ der grösste über $\Omega$ abelsche unverzweigte Körper. Wir fragen nach dem grössten sogar über $\mathrm{P}$ abelschen, über $\Omega$ unverzweigten Körper $\Gamma$; er heisse der Gcschlechterkörper von $\boldsymbol{\Omega}$.

1) T. Takagi, Über eine Theorie des relativ-Abelschen Zahlkörpers, Journ. Coll. Science, Tokyo 41 (1920), Art. 9, 1-131.

2) Cl. Chevalley, La théorie du corps de classes, Ann. of Math. 41 (1940), 394-418.

3) $H$. Hasse, Zum Hauptidealsatz der komplexen Multiplikation, Monatsh. Math. Phys. 38 (1931), 315-322. 
Wegen der Invarianz von $E$ beim erzeugenden Automorphismus $\tau$ von $\boldsymbol{\Omega} / \mathrm{P}$ ist K/P galoissch. Sei $\mathbb{3}$ die Galoisgruppe von K/P, und seien $\mathfrak{U}$,

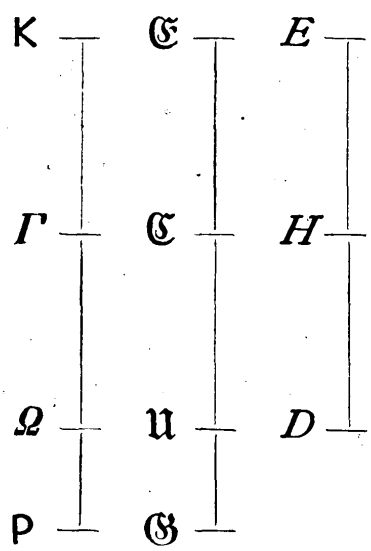
$\sqrt{5}$ die den Teilkörpern $\Omega, I$ zugeordneten Untergruppen. Dann ist $\mathfrak{U}$ ein Normalteiler von $\mathbb{S}$ vom Index 2 , und (C) ist die Kommutatorgruppe von (S).

Sei $D$ die Gruppe aller Divisoren a von $\boldsymbol{\Omega}$. Durch das Artin-Symbol

$$
\left(\frac{K / \Omega}{\mathfrak{a}}\right)=\sigma
$$

wird nach dem Artinschen Reziprozitätsgesetz die engere Divisorenklassengruppe $D / E$ von $\boldsymbol{\Omega}$ isomorph auf die Galoisgruppe $\mathfrak{u}$ von $K / \Omega$ bezogen, indem der Klasse $\mathfrak{a} E$ das Element $\sigma$ aus $\mathfrak{u}$ zugeordnet wird. Das Urbild $H$ von $\mathfrak{C}$ bei diesem Isomorphismus heisst das Hauptgeschlecht von $\boldsymbol{\Omega}$, und die Klassen von $D / H$ heissen die Geschlechtir von $\boldsymbol{\Omega}$, so dass also der Geschlechterkörper $\Gamma / \Omega$ der Klassenkörper zu der Geschlechtereinteilung in $\boldsymbol{\Omega}$ ist.

Die Struktur der Gruppe (S), und damit ihre Kommutatorgruppe $\mathbb{E}$, bestimmt sich nun folgendermassen.

Einerseits folgt aus der Isomorphismenregel für das Artin-Symbọl

$$
\left(\frac{K / \Omega}{\mathfrak{a}^{\tau}}\right)=\tau^{-1}\left(\frac{K / \Omega}{\mathfrak{a}}\right) \tau
$$

in Verbindung mit der Tatsache

$$
\left(\frac{K / \Omega}{\mathfrak{a}^{\mathfrak{\tau}}}\right)=\left(\frac{K / \Omega}{\mathfrak{a}}\right)^{-1} \quad\left(\text { da } \quad a a^{\mathfrak{\tau}}=\mathfrak{N}(\mathfrak{a}) \subset E\right),
$$

dass

$$
\tau^{-1} \sigma \tau=\sigma^{-1} \quad \text { für alle } \sigma \text { aus } \mathfrak{U}
$$

gilt.

Andrerseits sei $\mathfrak{P}$ ein nach dem Frobeniusschen Dichtigkeitssatz vorhandener Primdivisor von $K$ mit dem Frobenius-Symbol

$$
\left[\frac{K / P}{\mathfrak{P}}\right]=\tau,
$$

und seien $\mathfrak{p}, p$ die ihn enthaltenden Primdivisoren von $\Omega$, P. Nach der 
Reduktionsregel auf Teilkörper für das Frobènius-Symbol ist dann

$$
\left(\frac{\Omega / P}{p}\right)=\left[\frac{\Omega / P}{\mathfrak{P}}\right]=\mathfrak{u} \tau
$$

also $p$ in $\Omega$ träge; daher ist einerseits $\mathfrak{N}(\mathfrak{p})=p^{2}$, also nach dem Verschiebungssatz für das Frobenius-Symbol

$$
\left(\frac{\mathrm{K} / \Omega}{\mathfrak{p}}\right)=\left[\frac{\mathrm{K} / \Omega}{\mathfrak{P}}\right]=\left[\frac{\mathrm{K} / \mathrm{P}}{\mathfrak{P}}\right]^{2},
$$

und andrerseits is $\mathfrak{p} \simeq p \subset E$, also

$$
\left(\frac{K / \Omega}{\mathfrak{p}}\right)=1
$$

Zusammengenommen folgt

$$
\tau^{2}=1
$$

Nach (1), (2) ist $(\mathbb{S}$ die Erweiterung von $\mathfrak{U}$ mit der durch den Automorphismus , Übergang zum Reziproken“ erzeugten zyklischen Automorphismengruppe der Ordnung 2 und mit dem Faktorensystem 1 .

Die Kommutatorgruppe $\sqrt{5}$ von $(S)$ wird dann durch die Kommutatoren $\sigma^{-1} \tau^{-1} \sigma \tau=\sigma^{-2}$ mit $\sigma \subset \mathfrak{U}$ geliefert; es ist also

$$
\mathfrak{G}=\mathfrak{U}^{2} \text {. }
$$

Durch den Isomorphismus zwischen $\mathfrak{U}$ und $D / E$ ergibt sich hieraus der bekannte

Hauptsatz der Geschlechtertheorie. Das Hauptgeschlecht $H$ von $\boldsymbol{Q}$ besteht aus den Quadraten der Klassen von $D / E$, oder also aus den Quadraten der engeren Divisorenklassen von $\boldsymbol{\Omega}$.

Aus (3) folgt ferner, dass $\mathfrak{u} / \mathfrak{S}$ und damit auch $D / H$ abelsche Gruppen vom Exponenten 2 sind. Demnach gilt:

Der Geschlechterkörper $\Gamma$ von $\Omega$ ist aus quadratischen Körpern über P komponiert.

Als eine dieser Komponenten kann natürlich der Körpè $\boldsymbol{\Omega}$ gewählt werden.

2. Um den, Geschlechterkörper $\Gamma$ von $\Omega=\mathrm{P}(\sqrt{d})$ explizit zu bestimmen, gehen wir von der Tatsache aus, dass jede quadratische Körperdiskriminante (kurz: Diskriminante) $d$ eine eindeutige Zerlegung 


$$
d=p_{1}^{*} \ldots \ldots p_{r}^{*}
$$

in Primdiskriminanten $p_{*}{ }^{*}$, d.h. in Diskriminanten mit nur einem Primteiler $p_{i}$ besitzt. Als solche Primdiskriminanten kommen in Frage :

$$
\begin{array}{lll}
p^{*}=(-1)^{\frac{p-1}{2}} p & \text { für jede Primzahl } & p \neq 2, \\
p^{*}=-4, \pm 8 & \text { für die Pıimzahl } & p=2 .
\end{array}
$$

Die $d$ zusammensetzenden Primdiskriminanten $p_{i}^{*}$ gehören überdies zu verschiedenen Primzahlen $p_{i}$. Man erkennt sofort, dass jedes Teilprodukt $d^{\prime}$ aus den $p_{i}{ }^{*}$ ebenso wie sein komplementäres Teilprodukt $d^{\prime \prime}$ wieder eine Diskriminante ist, und dass umgekehrt jede Zerlegung

$$
d=d^{\prime} d^{\prime \prime}
$$

von $d$ in zwei Diskriminanten $d^{\prime}, d^{\prime \prime}$ aus der Primdiskriminanteñzerlegung durch Zusammenfassung zu Teilprodukten entsteht. Die Bezeichnung "Teiler " von $d$ sei nachstehend in diesem eingeschränkten Sinne verstanden.

Aus dem Zerlegungsgesetz für.quadratische Zahlkörper folgert man nun leicht :

Ein quadratischer Zahlkörper $\mathrm{P}\left(\sqrt{d^{\prime}}\right)$ licfert durch Komposition mit $\boldsymbol{\Omega}=\mathrm{P}(\boldsymbol{V} \bar{d})$ dann und nur dann einen über $\boldsymbol{\Omega}$ unverzzeigten Körper $\boldsymbol{\Omega}\left(\sqrt{\bar{d}^{\prime}}\right)$, also einen Teilkërper des Geschlechterkörpers $\Gamma$ von $\Omega$, zenn seine Diskriminante $d^{\prime}$ ein ",Teiler" von $d$ ist.

Auf die Ausführung des ganz einfachen Beweises, der auf der Doppeldarstellung

$$
\Omega\left(\sqrt{d^{\prime}}\right)=\Omega\left(\sqrt{d^{\prime \prime}}\right)
$$

beruht, können wir wohl hier verzichten.

Aus diesem Sachverhalt ergib̈t sich dann nach dem zuvor Gesagten ohne weiteres:

Der Geschlechterkörper $I$ von $\Omega=\mathrm{P}(\sqrt{d})$ ist aus den (unabhängigen) quadratischen Zahlkörpern $\mathrm{P}\left(\sqrt{p_{i}^{*}}\right)$ komponicrt, wo $p_{i}^{*}$ dic (vcrschiedenen) "Primtiler" von d sind.

Die Anzall der Geschlechter von $\Omega=\mathrm{P}(\sqrt{d})$ ist $2^{r-1}$, wor die Ansahl der verschiedenen Primteiler $p_{i}$ von $d$ ist.

3. Um die Einteilung der engeren Divisorenklassengruppe $D / E$ von 
$\boldsymbol{\Omega}$ in die Geschlechter (Klassen von $D / H$ ) durch Charaktere zu beschreiben, wenden wir den Verschiebungssatz der Klassenkörpertheorie auf die quadratischen Zahlkörper $\mathrm{P}\left(\sqrt{p_{i}{ }^{*}}\right)$ an. Nach ihm ist $\Omega\left(\sqrt{p_{i}^{*}}\right)$ Klassenkörper über $\Omega$ zur Gruppe der Divisoren a von $\Omega$ mit $\left(\frac{p_{i}^{*}}{\mathfrak{R}(\mathfrak{a})}\right)=1$, wobei man sich auf die $z u p_{i}{ }^{*}$ primen $\mathfrak{a}$ zu beschränken hat (während der genaue Führer jener Gruppe wegen der Unverzweigtheit 1 bezw. $p_{\infty}$ ist). Demgemäss wird das Hauptgeschlecht $H$ von $\boldsymbol{\Omega}$, als die Gruppe, zu der das Kompositum $\boldsymbol{I} \operatorname{der} \boldsymbol{\Omega}\left(\sqrt{p_{i}{ }^{*}}\right)$ Klassenkörper ist, im Bereich der zu $d$ primen Divisoren $\mathfrak{a}$ von $\boldsymbol{\Omega}$ durch die Bedingungen

$$
\left(\frac{p_{i}^{*}}{\mathfrak{N}(\mathfrak{a})}\right)=1 \quad(i=1, \cdots \cdots, r)
$$

charakterisiert. Da nach dem quadratischen Reziprozitätsgesetz

$$
\left(\frac{p_{i}^{*}}{\mathfrak{N}(\mathfrak{a})}\right)=\left(\frac{\mathfrak{R}(\mathfrak{a})}{p_{i}}\right)=\left(\frac{\mathfrak{N}(\mathfrak{a}), d}{p_{i}}\right)
$$

ist (für $p_{i}^{*}=-4$ unter Weglassung des Mittelgliedes), kann diese Charakterisierung von $H$, auch in der geläufigen, vorteilhafteren Form

$$
\left(\frac{\mathfrak{N}(\mathfrak{a}), d}{p_{i}}\right)=1 \quad(i=1, \cdots, r)
$$

geschrieben werden, in der die Beschränkung, auf zu $d$ prime a weggelassen werden kann (Erklärung von $H$ nach dem Führer 1 bezw. $p_{\infty}$ ). Demnach gilt :

Für beliebige Divisoren a von $\Omega$ charakterisiert das System der quadratischen Normsymbole $\left(\frac{\mathfrak{N}(\mathfrak{a}), d}{p_{i}}\right)$ für die Primteiler $p_{i}$ von d das Geschlecht, dem a angehört.

Da die Geschlechteranzahl $[D: H]=2^{r-1}$ ist, besteht zwischen diesen $r$ quadratischen Geschlechtscharakteren notwendig eine und nur eine Abhängigkeit. Sie wird durch die Produktformel für das quadratische Normsymbol gegeben, die sich hier auf

$$
\prod_{i=1}^{n}\left(\frac{\mathfrak{N}(\mathfrak{a}), d}{p_{i}}\right)=1
$$

reduziert.

4. In meiner oben zitierten Arbeit wird der Hauptdivisorsatz (dass 
nämlich in $K$-und sogar im Klassenkörper $K_{0}$ im weiteren Sinne zu $\boldsymbol{Q}$ jede Divisorenklasse von $\Omega$ in die Hauptklasse fällt) für einen imaginärquadratischen Grundkörper $\boldsymbol{\Omega}$ (wo $\mathrm{K}_{0}=\mathrm{K}$ ist) mit den analytischen Mitteln aus der Komplexen Multiplikation für alle diejenigen Divisorenklassen von $\Omega$ bewiesen, in welchen Divisoren a mit $\mathfrak{R}(\mathfrak{a}) \equiv 1$ mod. 12 vorkommen. ${ }^{4)}$ Die dort ausgesprochene Behauptung, dass dies für alle Divisorenklassen von $\boldsymbol{\Omega}$ zuträfe, ist nicht für jeden quadratischen Zahlkörper $\Omega=\mathrm{P}(\sqrt{d})$ richtig, wie Artin mir bald nach Erscheinen meiner Arbeit brieflich mitteilte.

Die vorstehend entwickelte Geschlechtertheorie erlaubt es, zu entscheiden, für welche Diskriminanten $d$ die fragliche Behauptung zutrifft. Zur Gruppe der Divisoren $\mathfrak{a}$ von $\Omega$ mit $\mathfrak{N}(\mathfrak{a}) \equiv 1$ mod. 12 gehört nämlich als Klassenkörper der Körper $\boldsymbol{Q}(\sqrt{-3}, \sqrt{-4})$ der 12-ten Einheitswurzeln über $\boldsymbol{S}$. Dann und nur dann, wenn dieser Körper fremd zum absoluten Klassenkörper $K$ ist, hat die Gruppe $\mathfrak{R}(\mathfrak{a}) \equiv 1$ mod. 12 mit jeder absoluten Divisorenklasse von $\Omega$ einen nicht leeren Durchschnitt. Dies ist aber nach der vorstehenden Theorie genau dann der Fall, wenn die Primdiskriminanten $-3,-4$ nicht als „Primteiler" von $d$ vorkommen.

Kommt nur -3 oder nur -4 als „Primteiler" von $d$ vor, so trifft fragliche Behauptung genau für die durch $\left(\frac{\mathfrak{R}(\mathfrak{a}), d}{3}\right)=1$ bezw. $\left(\frac{\mathfrak{R}(\mathfrak{a}), d}{2}\right)$ $=1$ charakterisierte Untergruppe $H_{3}$, bezw. $H_{4}$ vom Index 2 in $D$ zu; kommen -3 und -4 beide vor, so nur für die Untergruppe $H_{12}=H_{3} \cap H_{4}$ vom Index 4 mit bizyklischer Faktorgruppe $D / H_{12}$. Diese Untergruppen enthalten das Hauptgeschlecht $H$, setzen sich also aus vollen Geschlechtern zusammen.

Man kann nun in diesen Fällen die durch die Komplexe Multiplikation gelieferten Hauptdivisorbeziehungen in $K$ durch die Tatsache ergänzen, dass die Primteiler $\boldsymbol{p}_{\boldsymbol{b}}$ in $\boldsymbol{\Omega}$ der Diskriminantenprimteiler $p_{\boldsymbol{b}}$ trivialerweise Hauptdivisoren in $\Gamma$ und damit auch in $K$ sind. Demnach ist das in die Hauptklasse fallen für alle Klassen von $D / E$ gesichert, wenn den drei eben unterschiedenen Fällen entsprechend

in der Nebenklasse von $D / H_{3}$ ein Diskriminantenprimteiler $\mathfrak{p}_{6}$, in der Nebenklasse von $D / H_{4}$ ein Diskriminantenprimteiler $\mathfrak{p}_{i}$,

4) Dann kommen auch ganze, zu d prime Divisoren $m$ vor, wie a.a.O. schärfer zu verlangen ist. 
in zwei unabhängigen Klassen von $D / H_{12}$ ein Diskriminantenprimteilerpaar $\mathfrak{p}_{i}, \mathfrak{p}_{j}$

vorkommt.

Im ersten Falle ( -3 aber nicht -4 ,teilt“ $d$ ) sind ungünstig in diesem Sinne nur diejenigen $d$, für welche

$$
\left(\frac{p_{i}, d}{3}\right)=1 \quad(i=1, \cdots, r)
$$

ist; dies bedeutet, dass alle von 3 verschiedenen $p_{i} \equiv 1 \bmod .3$ sind (dann ist von selbst auch die Forderung für $p_{i}=3$ erfüllt).

Im zweiten Falle ( -4 aber nicht -3 ,teilt“ $d$ ) sind ungünstig nur diejenigen $d$, für welche

$$
\left(\frac{p_{i}, d}{2}\right)=1 \quad(i=1, \cdots, r)
$$

ist; dies bedeutet, dass alle von 2 verschiedenen $p_{i} \equiv 1 \bmod .4$ sind und überdies ihr Produkt $\equiv 1 \bmod .8$ ist,

Im dritten Falle ( -3 und -4 ,teilen“ $d$ ) sind diejenigen $d$ ungünstig, für welche bei jedem Paar $p_{i}, p_{j}$ die Determinante der $-1-$ Exponenten von

$$
\left(\begin{array}{ll}
\left(\frac{p_{i}, d}{3}\right) & \left(\frac{p_{i}, d}{2}\right) \\
\left(\frac{p_{j}, d}{3}\right) & \left(\frac{p_{j}, d}{2}\right)
\end{array}\right)
$$

gerade ist; explizit lässt sich das nur in einigermassen komplizierter Form ausdrücken.

Berlin, 14. August 1950. 